\begin{tabular}{l|l} 
REVISTA & $\begin{array}{l}\text { Revista Educación } \\
\text { ISSN: 0379-7082 } \\
\text { ISSN: 2215-2644 } \\
\text { revedu@ gmail.com } \\
\text { Universidad de Costa Rica } \\
\text { Costa Rica }\end{array}$
\end{tabular}

\title{
La perspectiva de género: una reivindicación necesaria en el ámbito educativo
}

\author{
Marrero Peniche, Gretel \\ La perspectiva de género: una reivindicación necesaria en el ámbito educativo \\ Revista Educación, vol. 43, núm. 2, 2019 \\ Universidad de Costa Rica, Costa Rica \\ Disponible en: http://www.redalyc.org/articulo.oa?id=44058158017 \\ DOI: https://doi.org/10.15517/revedu.v43i2.32426
}

Esta obra está bajo una Licencia Creative Commons Atribución-NoComercial-SinDerivar 3.0 Internacional. 


\title{
La perspectiva de género: una reivindicación necesaria en el ámbito educativo
}

The Need to Reclaim a Gender Perspective in Education

Gretel Marrero Peniche

Instituto Superior de Relaciones Internacionales (ISRI)

DOI: https://doi.org/10.15517/revedu.v43i2.32426
Redalyc: http://www.redalyc.org/articulo.oa?id=44058158017

"Raúl Roa García", Cuba

gretel@isri.minrex.gob.cu

iD http://orcid.org/ 0000-0001-8804-1576

\author{
Redalyc: hetp://wwwiredalyc.org/articulo.023id=44058158017
}

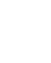


establecido como correcto o esperado para las mujeres en el mundo académico, motivo por el cual aún hoy se encuentra sustentada teórica y metodológicamente por los postulados feministas y la perspectiva de género.

Dicha plataforma teórico-metodológica constituye una especie de lupa gnoseológica con la cual todo el personal docente puede evaluar las discriminaciones de género que le rodean y, de hecho, las que una misma es capaz de generar si no se está alerta. El punto de partida de la reflexión que se propone surge de la propia experiencia de la autora. Se convierte entonces en piedra angular del análisis destacar la importancia de estudiar el medio educativo empleando la perspectiva de género, y algo más apremiante aun en la actualidad: implementar políticas públicas que permitan lograr los cambios necesarios en las prácticas educativas, y no para enunciar la existencia de problemas o discriminaciones.

Resultado de una relectura del ámbito educativo y gracias a los llamados de atención que ofrece la perspectiva de género, a la cual se debe la mirada crítica hacia toda forma -solapada o no- de discriminación solo por el hecho de ser mujeres u hombres en el medio educativo, se han ido acumulando una serie de preguntas como las siguientes: ¿qué tipo de hombres y mujeres estamos formando los que dedicamos nuestra vida a educar $^{[1]}$ ? ¿Cuál es el estado de desarrollo de la ciencia actualmente donde pretendemos que se inserten los y las estudiantes y cómo? Y lo más importante ¿de qué modelos de enseñanza somos hijos y a qué patrones respondemos hasta inconscientemente? Debido a estas y otras tantas interrogantes, se pretende dialogar con diversos postulados teórico-metodológicos que, a juicio de la autora de este trabajo, han sido determinantes para comprender la temática.

Un importante incentivo para el análisis que se presenta constituyó la Agenda 2030 para el Desarrollo Sostenible aprobada en el mes de septiembre del año 2015. Esta Agenda se propone como objetivo central la erradicación de la pobreza a nivel mundial para el año 2030, para lo cual fueron aprobados una serie de Objetivos por 193 Estados miembros de Naciones Unidas. La educación se presenta en "el Objetivo de Desarrollo Sostenible 4 (que consiste en) garantizar una educación inclusiva y equitativa de calidad y promover oportunidades de aprendizaje permanente para todos" (UNESCO, 2017, p. 3).

La Agenda 2030, en su espacio dedicado a la educación, parte del criterio de que la educación es capaz de impulsar el desarrollo y, de este modo, transformar vidas. Para que esto ocurra la propia educación debe cambiar: debe "estar abierta a todos [sin excepciones; debe] ser inclusiva y de buena calidad" (UNESCO, 2017 , p. 5). Estas condicionantes tienen lugar, en gran medida, debido a que el $14 \%$ de "los jóvenes y solo el $1 \%$ de las niñas más pobres completan la enseñanza secundaria en los países de bajos ingresos" (UNESCO, 2017, p. 5). Por tales razones, se hace énfasis en términos como inclusión, equidad e igualdad de género. Se convierte así el Objetivo 4.5: Igualdad entre los sexos e inclusión en uno de los más relevantes de la Agenda. La pretensión consiste en eliminar todas las disparidades de género existentes en el ámbito educativo, garantizando "el acceso igualitario a todos los niveles de enseñanza y formación profesional para las personas vulnerables, incluidas las personas con discapacidad, los pueblos indígenas y los niños en situaciones de vulnerabilidad" (UNESCO, 2017, p. 6).

Otro referente para el análisis fue presentado por la Organización para la Cooperación y Desarrollo Económico (OCDE) en su informe sobre el $A B C$ de Igualdad de Género en Educación: Aptitud, Comportamiento y Confianza (OCDE, 2015). En este caso, se pone el énfasis en analizar la educación bajo la perspectiva de género, no solo para declarar indicadores diferenciados según el sexo del estudiante, sino para buscar explicaciones que argumenten la existencia de esas diferencias. Se trata de trascender las descripciones sobre qué es lo diferente entre hombres y mujeres en el medio educativo y lograr comprender el por qué surgen dichas diferencias.

En dicho informe, la OCDE (2015) señala varios puntos neurálgicos para la comprensión de la temática. Se cuestiona cómo, teniendo en cuenta la existencia de igualdad de oportunidades en el acceso a la educación, existen diferencias en el alcance de los niveles educativos según el sexo del estudiante. Situación que apunta hacia la presencia de otras diferencias de género en la educación. Plantea que el contexto sociocultural actúa como condicionante del desarrollo educativo de los y las estudiantes. Denuncia además la existencia de un 
sistema simbólico sexista en el sistema educativo. Por tanto, cualquier cambio en las diferencias de género que se pretenda desarrollar debe tener en cuenta la relación sinérgica que debe establecerse entre varios sistemas, entre ellos, el familiar, el político el comunicativo, entre otros.

La selección del tema responde además a la consideración de que la "educación es el espacio favorable para construir el horizonte cultural de equidad de género y de diversidad sexual que permitiría tener las condiciones de posibilidad para una realización humana acorde con los intereses personales y sociales" (Barffusón, Revilla y Carrillo, 2010, p. 361). Por tanto, el objetivo central del trabajo que se presenta estará signado por: evaluar la importancia de implementar el pensamiento feminista y la perspectiva de género en el ámbito educativo, como herramientas indispensables para exponer y denunciar desigualdades de género.

\section{Parte I: Deudas gnoseológicas: de donde parte todo. Epistemología feminista [2] COMO GUÍA DEL ANÁLISIS.}

La comprensión del ámbito educativo como espacio de reproducción de desigualdades de género debe partir por la relación que se ha establecido históricamente entre la producción de conocimientos y el poder. Es decir, ¿qué temas han sido dignos de estudio y, por tanto, de transmisión en los textos educativos? Algunas de las principales respuestas que se han ofrecido al respecto se postulan teóricamente por la epistemología feminista.

Señala Diana Maffia que una de las propuestas epistemológicas feministas de mayor peso sobre el proceso de construcción de conocimientos, es la relación que se establece entre el conocimiento y el poder, y cómo esta misma relación de interdependencia es la que define quién, cómo y sobre qué temas se investiga. Lo cual significa que la dinámica sujeto-objeto ${ }^{[3]}$ de investigación pase obligatoriamente por la relación conocimiento-poder, toda vez que elementos como la objetividad del conocimiento se han validado -y aún hoy se validan - teniendo en cuenta el sujeto que lo produce. Entonces, la "legitimación de las pretensiones de conocimiento está íntimamente ligada con redes de dominación y de exclusión” (Maffia, 2007, p. 17).

Como complemento, la epistemología feminista lleva a cabo una desmitificación sobre los métodos de la ciencia. Concebidos hasta la década de 1960 como valorativamente neutros e independientes del contexto. Momento en el que los métodos científicos, como la observación, dejaron de concebirse como inocentes y comenzó a evidenciarse la influencia que sobre ellos ejercían los compromisos teóricos. Aunque en un primer momento lo anterior fue desarrollado por varios historiadores de la ciencia y filósofos de la ciencia de mentalidad histórica, se le atribuye a la epistemología feminista el hecho de situar el conocimiento científico, "es decir, mostrar la operación ideológica que supone esgrimir la noción de objetividad de la ciencia" (Fígari, 2011, citado por Fabbri, 2013, p. 39). En relación directa, las teorías del conocimiento también son puestas en tela de juicio por las corrientes de epistemología feminista, toda vez que comenzó a cuestionarse su supuesto valor universal.

Un excelente resumen sobre las críticas de los feminismos ${ }^{[4]}$ hacia la producción de conocimientos es presentado por Pilar Colás. La autora expone que

el Paradigma Feminista se centra en la crítica a la ausencia de mujeres en la ciencia, la defensa de una ciencia y una epistemología femenina o feminista, y el análisis de sesgos androcéntricos en los métodos y teorías científicas del pasado y del presente (Colás, 2003, p. 2).

Las críticas efectuadas a dicho sesgo androcéntrico de la investigación, presente en la ciencia tradicional, transitan en varios sentidos. Por un lado, apuntan hacia la discriminación de la mujer en "el acceso a la ciencia y, por otro, hacia la llamada mala ciencia y/o resultados sesgados por la posición subjetiva e interesada del investigador, condicionada por una posición y rol determinado" (Colás, 2003, p.3).

En consecuencia, el paradigma feminista critica la manera de hacer ciencia, lo cual queda resumido por Colás (2003) en tres planos: 
- la estructura organizativa y social de la ciencia

- la forma de hacer ciencia - pues no se tienen en cuenta temas claves en la investigación educativa-; la forma de hacer ciencia - pues no se tienen en cuenta temas claves en la investigación educativa-;

- la correspondencia entre valores sociales y valores de la ciencia (p. 3)

El primer plano señalado hace referencia básicamente "a nivel estructural en el escaso acceso de la mujer a la producción científica” (Colás, 2003, p. 3). Asimismo, alude a "las barreras que [le son impuestas a] las mujeres para ocupar puestos [de importancia o] decisivos en la ciencia y la tecnología; así como en la elaboración del discurso científico" (Colás, 2003, p. 3).

Sobre el segundo de los planos identificados por la autora, un elemento a destacar, lo cual lamentablemente se observa en no pocas oportunidades, es el hecho de que muchas investigaciones asumen la existencia de una sociedad única. Tal concepción implica hacer generalizaciones sobre sus participantes sin considerar que las realidades de hombres y mujeres -así como de grupos sociales- son diferentes y que, por tanto, exigen un tratamiento diferenciado.

El último plano que cita Colás es la "correspondencia entre valores sociales y valores de la ciencia. [Situándose] en un enfoque sociocultural de la ciencia, [expone cómo ese] estereotipo cultural [de una] ciencia dura, impersonal, racional" (Colás, 2003, p. 5), responde a una identidad de género masculina. Según esta autora, el discurso y el lenguaje científico constituyen herramientas que construyen y mantienen relaciones sociales asimétricas entre hombres y mujeres. Situación que se ha visto históricamente reflejada en la mayoría de los textos educativos.

Para ampliar sobre este particular resultan oportunas las ideas de Helen Longino (1993); Londa Schiebinger (1993) y Diana Maffia (2007). En tanto, coinciden en que es preciso concebir el conocimiento como producto de interacciones sociales, ello implica una comprensión del contexto de investigación y de las relaciones sociales que en él se desenvuelven ${ }^{[5]}$.

Otra reflexión de la epistemología feminista radica en la denuncia, "no solo al papel de la mujer como sujeto de producción de conocimientos, sino de los sesgos que el género imprime al producto, a la teoría científica” (Maffia, 2007, p. 18). En este sentido, Londa Schiebinger analiza cómo los fundadores de la ciencia moderna extrapolaron sus prejuicios - de género, raza, clase- a las investigaciones que elaboraban (Schiebinger, 1993, citado por Maffia, 2007). Situación que aun en la actualidad se observa en los procesos de construcción de conocimientos y que refuerza los valores culturales dominantes y androcéntricos.

Por tanto, la ciencia -en su sentido conceptual más amplio- no puede desprenderse de los sesgos o valores que se comparten en una comunidad científica. Como consecuencia, la persona que produce conocimientos debe reconocer que pertenece a un sistema social más amplio por lo cual está, inconsciente o conscientemente, condicionada por este.

Los planteamientos anteriores nos inducen a considerar que uno de los sesgos a los que se expone la producción de conocimientos es el sexista, lo cual constituye una denuncia de la epistemología feminista ${ }^{[6]}$ . Este precondicionamiento se evidencia no solo en la exclusión de la mujer en la construcción teórica del conocimiento, sino en la imagen que el producto del conocimiento ha generado de la naturaleza femenina y que contribuyó en gran medida al confinamiento social de la mujer -situación que el ámbito educativo históricamente ha reforzado-.

Con el objetivo de poner en evidencia tal situación, la epistemología feminista se ha encargado de estudiar el lenguaje de la ciencia. "La labor de las epistemólogas espontáneas fue, precisamente, la de encontrar respuestas a la ausencia sistemática de las mujeres en los textos científicos” (León, 2011, p. 159), pero además, la mirada feminista se encargó de sacar a la luz la falsa neutralidad del discurso científico.

Según planteamientos feministas - con los que se concuerda totalmente- el lenguaje científico no ha sido en absoluto neutral, pues "se filtran en él valores y no es meramente descriptivo" (Maffia, 2007, p. 20). La ideología patriarcal subyace en el lenguaje de la ciencia escondida bajo una supuesta neutralidad. Por tanto, 
resulta importante la inclusión del concepto de género en la década de los 70 por parte de las feministas, no como referencia a una definición biológica, sino como la construcción de pautas sociales que moldean en cada sociedad una ideología de género.

Una frase muy oportuna que debe incidir en todos los que, de una forma $u$ otra, dedicamos nuestro quehacer diario a investigar o a educar es esbozada por María Elena León: "las epistemologías feministas deben servir como mediadoras en un proceso en el que se requiere de crítica para poder distinguir una nueva forma de búsqueda del saber" (León, 2011, p. 157).

A modo de resumen, las reflexiones epistemológicas feministas ${ }^{[7]}$ cuestionan el marco conceptual en el que se sostiene el modo tradicional de producción de la ciencia. El sujeto y el objeto de la investigación son analizados como resultado de raciocinios patriarcales y androcéntricos, así como los procesos de construcción de conocimientos.

La perspectiva feminista -aunque no solo esta- introduce problemas de investigación novedosos; cambiando así la forma de concebir la metodología de la investigación. Incorpora en el análisis las experiencias de las mujeres, pero no experiencias fuera de contexto, sino todo lo contrario. Desborda las categorías sujetoobjeto, toda vez que el investigador debe dejar su posición privilegiada y asumirse también como un sujeto histórico con prejuicios e ideas preconcebidas. Privilegia además la perspectiva histórica y el análisis de las relaciones de poder. Elementos todos que alcanzan un claro reflejo en las prácticas educativas.

Lo anterior no significa en lo absoluto la inexistencia de conflictos al interior del pensamiento feminista. De hecho, como plantea Mary Goldsmith en Feminismo e investigación social. Nadando en aguas revueltas, no hablamos de una discusión que transita en direcciones unívocas.

Por tales motivos, se plantea que:

Evidentemente no hay acuerdo entre las feministas acerca de en qué consiste el conocimiento o cómo se genera, ni en cómo se construye y se aborda un problema de investigación, ni acerca de si puede haber criterios para evaluar la validez o no de los resultados. Tampoco hay consenso sobre la existencia o no de una epistemología, metodología o método feminista (Goldsmith, 1998, p. 43).

\section{No obstante, Teresita De Barbieri en Acerca de las propuestas metodológicas feministas sugiere:}

En otras palabras, no hay una ética particular feminista ni normas que caractericen la investigación que hacen las feministas. Lo que existen son principios fundamentales de respeto y salvaguarda de la libertad y la dignidad humanas, que es necesario adecuar en todas las actividades que realizamos (De Barbieri, 1998, p. 125).

Se considera que en este último elemento radica uno de los principales aportes del pensamiento feminista ${ }^{[8]}$ : su insistencia en la defensa de la dignidad humana en cualquier actividad que se organice. De este modo, hace un llamado a no segregar o discriminar por motivos de sexo, raza, etnia u otros, a respetar la diversidad y a reconocernos dentro de ella.

\section{Parte II: El espacio educativo: Centro de la Reflexión.}

La primera interrogante que pudiera plantearse en esta segunda parte de la reflexión sería ¿cómo aplicar los referentes teóricos feministas esbozados con anterioridad para comprender el accionar histórico de diferencias de género en el ámbito educativo? $\mathrm{O}$ ¿dónde radican sus principales aportes?

Si se analiza el ámbito educativo desde una perspectiva histórica, podría observarse cómo, generalmente, la mujer ha sido ubicada en una posición inferior al hombre. Primero, por el denegado acceso que tenían las mujeres a la educación; luego de lograrlo, era muy limitado el campo de conocimientos al que podían aspirar

[9]. Con posterioridad, y tras muchas batallas feministas, la mujer accedió totalmente a la educación, pero sus conquistas no aparecían en los libros de texto, era invisible. Por tanto, en la educación, esa ha sido la principal 
tarea de la crítica feminista: hacer visibles a las mujeres y exponerlas, no como víctimas sino como iguales en el proceso de creación de conocimientos.

Resultan oportunos los planteamientos de Magdalena Valdivieso al respecto. La autora argumenta que, "utilizar la perspectiva de género implica reconocer... que una cosa es la diferencia sexual y otra cosa son las atribuciones, ideas, representaciones y prescripciones sociales que se construyen tomando como referencia a esa diferencia sexual" (Valdivieso, 2007, p. 14). Sus ideas conducen a considerar que, los mismos elementos que se imponen para llevar a cabo un estudio con perspectiva de género, se ajustan para comprender el papel que juega el personal docente al impartir los contenidos en el salón de clases.

Se argumenta a continuación con mayor detenimiento. Desde los inicios de su etapa formativa, la autora del presente ensayo, tuvo la oportunidad de acercarse a investigadores cubanos que aplicaban en sus estudios la perspectiva de género y los aportes epistemológicos del feminismo, lo cual llamó en buena medida su atención pues no era - ni es aun hoy ${ }^{[10]}$ - algo generalizado en el medio académico. Entre esos autores se encontraba la Dra. Marta Núñez Sarmiento -a quien la autora debe sus primeros pasos en el tema-.

Lo que interesa ahora es ver cómo se traducen las recomendaciones de la perspectiva de género en la labor del personal docente, así como de investigadoras e investigadores del ámbito educativo. La primera tarea sería definir qué puede entenderse por perspectiva de género [11]. Acorde a investigaciones de Núñez Sarmiento, una buena parte de científicos sociales no dedican amplios espacios a conceptualizar teórica y metodológicamente qué entienden por perspectiva de género. Diversos estudiosos del tema en Cuba, conciben el enfoque de género como un concepto o concepción metodológica y epistemológica multidefinible, esto se debe a que la realidad que estudian es ontológicamente diversa; además, al acercarse a esta realidad lo hacen desde sus profesiones y experiencias vitales (Núñez, 2001).

Por tales razones, la definición de perspectiva de género está abierta a futuras incorporaciones de conocimientos y se encuentra en un proceso constante de construcción en la medida en que cada investigador amplía su cultura sobre el tema. "Es una concepción esencialmente antidogmática (...) no acepta verdades establecidas de antemano. Quien practique este enfoque (...) no admite que a su pensamiento le impongan mecanismos de poder" (Núñez, 2001, p. 6). Pensamiento este último que alcanzaría un feliz reflejo en lo que podríamos considerar como buenas prácticas educativas ${ }^{[12]}$.

Si se analizan los planteamientos recién expuestos desde una mirada más abarcadora, se podría percibir que coincide con el rol del sistema educativo, al menos con su deber ser. Las condiciones sociales actuales exigen cada día más que el aula se convierta en un espacio antidogmático, donde profesores, profesoras y estudiantes construyan juntos el conocimiento, tomando los paradigmas teórico-metodológicos que les han precedido pero incorporando experiencias propias y ajustándolas a un contexto histórico-social concreto - punto este último que señala el enfoque de género no debemos perder de vista ${ }^{[13]}$.

De forma similar sucede con los mecanismos de poder que intentan imponer los métodos de enseñanza herederos de una cultura androcéntrica, donde el rol de la mujer en la historia de la ciencia pasa desapercibido. Situación que se perpetúa si al interior de las aulas también se establecen jerarquías en función del sexo de los estudiantes. O sea, que es posible identificar una doble discriminación de género: por un lado, los conocimientos que se imparten aun desde dogmas y contenidos masculinizados, resultado de toda una cultura de la enseñanza androcéntrica; y, por otro, las propias fallas del personal docente al impartir este contenido si no se cuenta con una preparación teórico-metodológica en lo que a perspectiva de género se refiere.

Sobre este particular, resultó nuevamente de gran utilidad el texto de Pilar Colás Investigación educativa y crítica feminista en relación con las agendas de investigación educativa crítica desde una perspectiva de género, sobre todo en la formación del personal docente e investigativo. Se concuerda plenamente con la autora al plantear que la "identificación y generación de conocimientos científicos en las relaciones de género se traducirá en cambios en las prácticas educativas” (Colás, 2003, p. 10).

En otro sentido, pero en estrecha relación con las ideas anteriores, un aspecto que merece mayor detenimiento es la visión de la perspectiva de género sobre las relaciones de poder que son establecidas entre 
hombres y mujeres. Principalmente porque implica que toda problemática que se estudie tiene que tomar en cuenta cómo se han articulado las relaciones sociales de lo masculino y lo femenino en cada contexto determinado ${ }^{[14]}$.

En el ámbito educativo podemos encontrar diversas manifestaciones de dichas relaciones de poder que son puestas en evidencia a través del pensamiento feminista y de la perspectiva de género. Entre ellas:

- La tradición de la bibliografía escolar de mostrar al hombre en el ámbito público y reservar la exclusividad del espacio privado para la mujer -discriminación de género en la literatura escolar-;

- la diferencia en las selecciones profesionales hacia las que se encaminan a mujeres y hombres, donde estos últimos continúan prevaleciendo en carreras técnicas;

- la existencia de un currículo oculto que funciona más eficazmente que el oficial, a través del cual docentes y otros estudiantes encasillan a los otros en función de, entre otras cosas ${ }^{[15]}$, el pertenecer a uno u otro sexo;

- la carencia de definiciones curriculares que inserten programas dedicados a estudios de género y cómo se articulan con el resto de los contenidos estudiados.

Los contenidos curriculares reflejan también el protagonismo del arquetipo masculino y se caracterizan por la invisibilidad de las mujeres, por el tratamiento discriminatorio de lo femenino y por la ausencia de todos aquellos aspectos vinculados al mundo privado, a la vida cotidiana, a las relaciones personales y a la esfera doméstica (Barffusón et al., 2010, p. 374).

- el hecho de que no siempre el total acceso a la educación de la mujer se traduce en un tratamiento igualitario en cuanto a política laboral se refiere, lo que ha sido identificado también como techo de cristal en tanto el acceso limitado a ascender en puestos de trabajo solo por el hecho de ser mujeres;

- además, entre tantos otros ejemplos que podrían citarse:

Tampoco hemos superado que los estudios de género sean una especialización femenina. Difícilmente encontramos que se incorpore el género y los intereses de las mujeres en las investigaciones generales que se realizan (Valdivieso, 2007, p. 9).

Para comprender el accionar del currículo oculto, mencionado en el tercer punto de la lista precedente, es preciso considerar que la educación, tanto formal como informal, no solo ha transmitido el orden social existente, sino que ha reproducido patrones culturales sobre el significado de ser hombre o ser mujer en cada sociedad. Elementos que aún hoy influyen, por ejemplo, cuando se hace mención a los estudios estereotipados según el sexo del estudiante, por lo que muchas de las titulaciones universitarias se encuentran ya definidas socialmente.

Sobre el tema, Francesca Gargallo cuestiona lo siguiente:

Cómo la educación formal puede dejar de ser un instrumento de repetición, asimilación y naturalización de pautas sexistas, si los sujetos de las mismas no son preconcebidos como neutros, que ocultan una naturalizada asignación de roles genéricos, sino como personas sexuadas, con derechos, presencias, intereses, historias individuales y colectivas, y aportes propios al conjunto de la sociedad (Gargallo, 2008, p. 18).

Tales planteamientos alcanzan especial relevancia en la actualidad pues, como parte del accionar del currículo oculto, muchas veces el personal docente encasilla ${ }^{[16]}$ a los y las estudiantes en determinadas ramas del conocimiento solo por el hecho de ser hombres o mujeres. Aunque este encasillamiento en ocasiones ocurre de forma inconsciente por parte de profesores y profesoras - pues ya la sociedad ha definido para qué tipo de estudios estamos dispuestos y dispuestas- no es menos cierto que ejerce una influencia en el estudiantado, definiendo así sus aspiraciones educativas en el futuro.

Esta situación tiene lugar precisamente porque la existencia de un currículo oculto se manifiesta:

En gestos, omisiones, selecciones sesgadas de materias, miradas de aprobación o reprobación que reflejan la posición de las y los docentes sobre los temas en que se asientan las desigualdades sociales, las prácticas culturales hegemónicas y los conflictos de género en las aulas (Gargallo, 2008, p. 21). 
En consecuencia, se considera que la principal herramienta que brinda la crítica feminista al análisis de la educación como espacio de reproducción de desigualdades de género, específicamente el feminismo de la diferencia, es que constantemente se interroga sobre el rol de la mujer en el ámbito educativo: como alumnas, profesoras, madres. Pero no como seres discriminados sino como sujetos pensantes, independientes, autónomos, y por tanto capaces de producir conocimientos sin límites preestablecidos.

En el caso específico del currículo oculto, los presupuestos feministas hacen visibles las prácticas educativas que evidencian su accionar, por lo cual también ofrecen vías para combatirlo: prácticas educativas inclusivas, desde el reconocimiento de la diferencia, que se traduzcan en equidad de género.

Como propuesta teórica y metodológica, el feminismo permite que el personal docente tome conciencia de que el currículo oculto:

Responde a los supuestos indecibles de la transmisión de prejuicios, y se adscribe a la repetición por parte de las y los docentes, de saberes que no se expresan en una práctica crítica de los mismos, sino en la aceptación-repetición sin examen de lo aprendido en estudios tendientes únicamente a la titulación, entendida esta, como un trámite para la adquisición de un instrumento de acceso al trabajo (Gargallo, 2008, p. 21).

Por estos y muchos otros motivos, aplicar la perspectiva de género en el salón de clases contribuye a poner en evidencia las represiones que se ejercen desde el poder, tanto a nivel social como personal. Lo que implica "dirigir la atención hacia el modo en que la escuela establece la relación entre los sexos y cómo prepara a ambos de diferente manera para sus respectivos destinos" (Barffusón et al., 2010, p. 374).

No se trata de intentar construir desde las aulas o la academia un mundo de mujeres, como en varias ocasiones se ha malinterpretado a la teoría feminista. El objetivo radica en brindarle equitativa importancia, en la impartición de contenidos y en los estudios desde las ciencias sociales, a mujeres y hombres, tomando como criterio básico la diversidad de experiencias por las que históricamente han atravesado.

En un sentido generalizador, es preciso comprender que "los sujetos y objetos de conocimiento de la investigación feminista son: la vida y práctica de las mujeres y de los hombres implicados en relaciones de género" (Gordillo, 2004, p. 6). O como planteara Kamala Viswesaran, "habría que estudiar a la mujer, pero en relación con los hombres y en relación con otras mujeres" (Viswesaran, 1994, p. 20, citado por Goldsmith, 1998, p. 50).

En correspondencia, los que se desempeñan en el medio educativo deben tener presentes las trampas metodológicas a las que se exponen, incluso de manera inconsciente. Tal es el caso de la impartición de asignaturas como Metodología de la Investigación Social. En ese sentido, resulta oportuno hacer referencia a las técnicas de investigación, pues el pensamiento feminista propone un cambio en la interpretación ${ }^{[17]}$ de las mismas (Gordillo, 2004).

Con el propósito de ejemplificar el planteamiento anterior sería oportuno retomar una idea esbozada previamente: bajo la perspectiva de género, la observación no es neutra, nunca lo ha sido, pues en ella participa la ideología del investigador, ¿qué aspectos, sujetos y momentos privilegia para la observación? Al respecto, Eli Bartra expone: "no existe la observación neutra. Ni siquiera existe la observación objetiva en el sentido de que todos los individuos que observan un proceso deben hacerlo de la misma manera en aras, supuestamente, de obtener un resultado objetivo" (Bartra, 1998, p. 6).

Por tanto, se proponen técnicas más participativas -herederas en gran medida del pensamiento feminista-. Bajo tal óptica, el conocimiento no se construye de forma lineal sino más bien como una espiral, con adelantos y retrocesos, contando con las experiencias de los y las investigados/as y del propio grupo de investigadores.

Estos y otros elementos deben ser tenidos en cuenta al impartirle al alumnado, más que las técnicas que se emplean en la investigación social, el cómo se emplean, de este modo se contribuye a evitar investigaciones de pre y postgrado caracterizadas por análisis mecanicistas y androcéntricos.

Pero, en última instancia, no resulta constructivo dedicarse a culpar a estudiantes o docentes. Se debe insistir en la importancia de marcar la diferencia a través de la implementación de la perspectiva de género 
sustentada en los postulados teórico-prácticos feministas. Además, es preciso hacer énfasis en aspectos como la idoneidad de determinadas técnicas de investigación según la temática a indagar.

Se concuerda entonces con Teresita De Barbieri al plantear que de lo que "se trata en la gran mayoría de las investigaciones que se realizan cuando se introduce la dimensión de género, es de aumentar la precisión de las descripciones y las explicaciones de las sociedades y de determinados conjuntos sociales" (De Barbieri, 1998, p. 120).

En consecuencia, las técnicas cualitativas son más adecuadas en la investigación feminista por el carácter subjetivo, la "implicación personal, la sensibilidad a los fenómenos complejos y únicos, el conocimiento contextual, la perspectiva individual y la posibilidad de crear un intercambio de experiencia entre investigador e investigado" (Gordillo, 2004, p. 9).

Sucede que estas cualidades han sido empleadas por las miradas androcéntricas para denotar a este tipo de investigaciones como no válidas o poco cientificas.

Otro de los elementos a considerar, al hablar del pensamiento feminista y de la perspectiva de género en la educación, resultan los planteamientos de Barffusón y otros autores, sobre la necesidad de continuar trabajando en aras de lograr "un cambio cultural profundo" (Barffusón et al., 2010, p. 359) que incida en el sector educativo. No se trata solo de educar con perspectiva de género, sino de convertir esa educación en políticas públicas que permitan la transmisión de conocimientos basados en la equidad como principio rector.

La historia de la educación heredada, tanto en su dimensión formal como en la informal, "ha contribuido de manera significativa a la creación jerarquizada de las relaciones intergenéricas" (Barffusón et ál., 2010, p. 360). Por ello, se impone un cambio estructural en ambas dimensiones, cuyo principal objetivo es extrapolar ese cambio a la vida cotidiana, permitiendo así la prevalencia de nuevos valores universales, ya no de los masculinos.

Para lograr tal objetivo, se impone comprender a cabalidad el terreno en el que se desenvuelve cada docente, en tanto la educación tiene una doble cara: actúa como "instrumento de transformación tanto como instrumento de reproducción del orden” social (González, 2005, citado por Ungo, 2007, p. 9). Por lo que, a consideración de Urania Ungo, "los procesos educativos pueden actualizarse para acompañar del mejor modo los cambios progresivos que permitan ir cerrando la brecha de la desigualdad" (Ungo, 2007, p. 9).

Recordemos además que,

El método educativo del sistema dominante es en realidad un mecanismo de adiestramiento a la obediencia de pautas de comportamiento y de aprendizaje incuestionables, que se sostiene en la identificación de lo hegemónico con lo científico, lo racional, lo masculino, lo desarrollado (Gargallo, 2008, p. 23).

Por tanto, se considera que las ciencias sociales han cometido en diversas ocasiones el error de analizar procesos y fenómenos sociales de forma desarticulada e independiente, sin observar relaciones lógicas que pueden establecerse. Por ello, en la actualidad se insiste en la necesidad de desarrollar estudios multi, inter y transdisciplinares, que logren esa articulación necesaria que permita analizar los temas de investigación desde miradas más holísticas.

En palabras de Magdalena Valdivieso,

Lo deseable es la producción de un conocimiento útil para el desarrollo y la preservación de la vida en el planeta, que en los distintos ámbitos genere ideas y propuestas en las que todas y todos podamos reconocernos en nuestras singularidades y al mismo tiempo confundirnos con los otros y otras sujetos y sujetas como parte de una humanidad diversa y plural (Valdivieso, 2007, p. 19).

La inclusión del párrafo anterior responde a la consideración de que constituye otro error separar los estudios sobre el ámbito educativo de aquellos relacionados con los procesos de producción de conocimientos, es decir, sobre la academia. La relación que mantienen ambos es tan estrecha, que todos los esfuerzos por introducir la perspectiva de género en las investigaciones efectuadas van a tener una influencia 
directa en la forma en la que se imparten los conocimientos. Lo anterior ocurre, principalmente, porque en la actualidad se combina en muchas ocasiones la actividad docente con la investigativa.

Autoras como Urania Ungo han alertado sobre procesos que retan a la perspectiva de género en el ámbito educativo como: el apoyo de varios Estados a la educación privada como sectores de excelencia para formar gobernantes que se enfrenta a otro sector menos competitivo, público, destinado a formar a los gobernados ${ }^{[18]}$ . O el hecho de que el "acceso a la educación ya no sea un obstáculo para la mujer pero sí el ascenso, entendido como movilidad hacia arriba y no solo como expansión cuantitativa y horizontal” (Ungo, 2007, p. 3).

A lo que agregaría Barffusón, que ser mujer u hombre continúa siendo determinante en el proceso educativo, en "el de profesionalización y, sobre todo, en el acceso a la educación universitaria y su posterior inserción y promoción laboral” (Barffusón et ál., 2010, p. 372).

Como resultado de ese proceso de adiestramiento que se recibe en el ámbito educativo -formal e informalacorde a cada sexo, basta analizar quiénes han sido por mayoría abrumadora los gobernantes a nivel mundial. Aunque hay que reconocer la aparición de mujeres valiosísimas en la política internacional habría que indagar hasta dónde han sido las barreras a superar, solo o principalmente, por el hecho de ser mujeres.

Es preciso además, declarar todos los medios que abarca la transmisión de conocimientos y pautas de comportamiento fuera de las puertas del ámbito educativo formal. Entre ellos: la "publicidad, los medios masivos de comunicación, la definición estética, la propuesta de un único tipo de vida de familia, siempre más reducida, una especie de pareja de ensayo, sin contacto con afectos externos que la liberen de su encierro" (Gargallo, 2008, p. 24).

Uno de los últimos aspectos que se quisiera señalar, se encuentra estrechamente relacionado con la necesidad de abandonar el dogmatismo en el empleo del pensamiento feminista y de la perspectiva de género para evidenciar desigualdades al interior del ámbito educativo. Al respecto, resultó muy acertada la siguiente idea planteada por Barffusón y otros autores: el "feminismo se proyecta críticamente hacia la educación a la cual se cree neutra y que sin embargo es totalmente segregada y sexista, esencialmente masculina” (Barffusón et ál., 2010, p. 363).

Esta frase induce a analizar el ámbito educativo en dependencia del contexto histórico-social concreto, pues los avances y retrocesos han sido diversos en cada región o país. Por tanto, la utilidad de términos definidos por la crítica feminista - como patriarcado, androcentrismo y falogocentrismo- que ponen en tela de juicio el ideal masculino como patrón de conocimiento válido estará en dependencia del uso correcto o no que se le otorgue, en tanto cada sociedad ha experimentado de forma diversa las discriminaciones hacia la mujer en la educación. Tener en cuenta este presupuesto posibilita idear y aplicar políticas públicas acordes al espacio que se pretende transformar y no crear líneas generalizadas que no cumplan el cometido para el cual fueron diseñadas.

Si una realidad se debe tener presente es que el acceso de las mujeres a la educación formal no es suficiente. Si bien en varios países de América Latina y el Caribe ${ }^{[19]}$ se ha logrado una mayor matrícula femenina en los espacios educativos formales, eliminando de este modo la más antigua de las discriminaciones de género ${ }^{[20]}$, no se ha logrado aún identificar zonas más solapadas de discriminación. Momento actual que Urania Ungo define "como cambios, avances y contradicciones" (Ungo, 2007, p. 3 ).

Una de esas zonas solapadas de discriminación a las que hace referencia el párrafo anterior responde a la relación que se establece entre las condiciones sociales desfavorables que enfrentan numerosas mujeres en países de América Latina y cómo esta situación incide, no solo en el escaso acceso al sistema educativo formal, sino en el hecho de no hallarse representadas en él. Pareciera que las herencias culturales o étnicas de las cuales proceden muchas mujeres y hombres ${ }^{[21]}$ latinoamericanos no existen, pues el sistema educativo las obvia para transmitir valores promovidos como universales ${ }^{[22]}$.

Por tales motivos, no se debe estar conforme con la inclusión numérica de mujeres en el sistema educativo formal, sino considerar que el proceso conlleva análisis puntuales. Es preciso estudiar cada región y contexto 
específicos para diseñar políticas efectivas, analizando de forma íntegra la situación social en las que se desenvuelven las mujeres, que muchas veces condiciona -hasta al interior de una familia- su acceso a la educación $^{[23]}$.

Son, profesoras y profesores, en gran medida responsables de los futuros profesionales que ayudan a formar, junto a la familia y a otros componentes sociales. Tienen en su poder valiosas herramientas de trabajo como el pensamiento feminista y la perspectiva de género- para sacar a la luz y denunciar comportamientos discriminatorios.

Son, además, resultado de un tiempo histórico y de una formación no siempre sustentada en valores de justicia social. Queda, por todos lo que dedicamos nuestra vida laboral -y personal- a la docencia, descubrir, estudiar y emplear alternativas en el quehacer cotidiano, que es la educación de otros que nos juzgarán y juzgarán a las sociedades donde viven en la misma medida en la que seamos capaces de educarlos en valores de bien común y de justicia social. No debe temerse a la deconstrucción de conocimientos preestablecidos que discriminen a sectores sociales por motivos de género, raza, etnia, entre otros ${ }^{[24]}$. Por el contrario, se debe ser consciente de la existencia de alternativas, como la "coeducación consciente y explícita de la igualdad de roles" (Barffusón et al., 2010, p. 363).

Aunque en algunos países el término coeducación ${ }^{[25]}$ ha sido sustituido por el de educación para la igualdad -como en España y México en la década de los 90-, se considera que lo más trascendente no radica en el debate conceptual entre ambos, sino en la efectividad de reconocer que es necesario un cambio en la concepción del espacio educativo. De tal modo que se construya como un lugar ideal para reconocer la diversidad, respetarla y transmitirla. Es preciso crear conciencia en el estudiantado de que han existido y existen diferencias entre hombres, mujeres, etnias, clases sociales, entre otros- en los contenidos educativos y en la forma de impartición de los mismos. Solo así seremos capaces de lograr en el ámbito educativo ese cambio cultural profundo al que varios autores, sustentados en el pensamiento feminista y en la perspectiva de género, han hecho referencia.

Resulta pertinente resumir lo anterior en la siguiente frase:

La incorporación de la perspectiva de género en la educación abre el complejo panorama de la teoría feminista. El campo educativo es un espacio idóneo para llevar a cabo parte de la transformación social que está en la base de su planteamiento: denunciar el sesgo del falogocentrismo en el ámbito educativo y ofrecer iguales alternativas (Barffusón et al., 2010, p. 358).

\section{CONSIDERACIONES FINALES:}

La epistemología feminista y la perspectiva de género pueden concebirse como herramientas teóricometodológicas de gran utilidad para el análisis de las prácticas docentes, en tanto permiten evidenciar las desigualdades de género presentes en el sector educacional.

Algunos de los elementos a los cuales alude se encuentran resumidos en: la invisibilidad de la mujer en los libros de texto básicos del sistema educacional -o una visibilidad reflejada en el antagonismo de los espacios público-privado, en tanto el primero reservado para los hombres y el segundo para las mujeres como algo natural-; la metodología de la investigación de carácter androcéntrico que reproduce la situación desigual de la mujer en la producción de conocimientos; la situación de invisibilidad de la mujer en la historia de la ciencia; la existencia de un currículo oculto y de prácticas docentes que encasillan al alumnado según el sexo y no las capacidades intelectuales y personales, entre tantos otros elementos.

A pesar de los avances logrados en materia de educación a nivel internacional, en tanto acceso igualitario de niñas y niños en casi todos los países, quedan pendientes prácticas educativas las cuales permitan reducir las diferencias de género al interior del salón de clases.

En tal sentido, deben incluirse prácticas educativas que faculten aprender de la experiencia e incorporar ese aprendizaje en el actuar cotidiano del personal docente. No se trata de pretender generalizaciones 
esquemáticas que no aporten aprendizaje en relación con el contexto social e histórico en que se vive, ni de importar modelos exactos cuando se habla de buenas prácticas. Se trata de adaptar al contexto las experiencias docentes exitosas. Se debe, a través del respeto, la reciprocidad y la cooperación, fomentar en los alumnos un aprendizaje activo, de raciocinio en vez de memorización dogmática.

La relación que se establece entre la epistemología feminista, la perspectiva de género y las buenas prácticas educativas apunta hacia la manera en que cada estudiante se adapta al proceso de enseñanza, asumiendo de manera individual los contenidos que se imparten. Por ello, se precisa una atención personalizada, así como reconocer ese currículo oculto que opera de forma solapada y que estereotipa al alumnado por motivos de género.

La función del docente debe ir más allá de la impartición del contenido, debe buscar la motivación y la creatividad del estudiantado, mostrando la amplitud gnoseológica del camino a seguir sin imposiciones esquemáticas. Se debe reconocer la existencia de una convivencia intercultural la cual se debe aceptar y respetar, transmitiendo aquellos contenidos que contribuyan a fomentar la equidad de género.

Las buenas prácticas docentes deben ser entendidas como el deber ser de la educación. Deben ir acompañadas por la experiencia del cuerpo docente, a la vez que se introducen cambios necesarios con el propósito de lograr una educación inclusiva y de equidad de género. En sentido general, las buenas prácticas deben ser innovadoras, proponiendo soluciones creativas a las problemáticas actuales en cada sociedad. Por tanto, efectivas, lo que significa que tengan un reflejo tangible en la superación de dificultades. Deben ser además sostenibles y replicables, lo cual implica que puedan mantenerse en el tiempo, producir efectos duraderos y tener la capacidad de aplicación en otros lugares. En el sector educacional podrá concebirse como una buena práctica aquella iniciativa, política o modelo de actuación, que permita el mejoramiento de los procesos docentes, así como los resultados educativos de los alumnos ${ }^{[26]}$.

La coeducación, entendida no solo como la educación conjunta de niños y niñas, hombres y mujeres, debe promover valores y actitudes igualitarios sin que medien diferencias de género. Cada docente debe proponerse educar desde modelos culturales que contribuyan a eliminar diferencias según el sexo del estudiantado. Educar, por tanto, reconociendo las diferencias que existen y, sobre esa base, propiciar la equidad.

\section{REFERENCIAS}

Acker, S. (1995). Género y educación. Reflexiones sociológicas sobre mujeres, enseñanza y feminismo. España: Narcea

Barffusón, R., Revilla, J. A. y Carrillo, C. D. (2010). Aportes feministas a la educación. Enseñanza e investigación en psicología, 15 (2), 357-376

Bartra, E. (1998). Reflexiones metodológicas. En E. Bartra (Comp.) Debates en torno a una metodología feminista (pp. 141-158.). México: Universidad Autónoma Metropolitana-Unidad Xochimilco

Colás, P. (2003). Investigación educativa y crítica feminista. Agora digital, 6, 1-14 Recuperado de: https://bit.ly/30 jbuTN

De Barbieri, T. (1998). Acerca de las propuestas metodológicas feministas. En E. Bartra (Comp.) Debates en tomo a una metodologí feminista (pp. 103-140.). México: Universidad Autónoma Metropolitana-Unidad Xochimilco

Fabbri, L. (2013). Masculinidad y producción de conocimiento no androcéntrico. Interpelaciones de la epistemología feminista. Revista Sujeto, Subjetividad y Cultura, 5, 36-44

Gargallo, F. (2008). El feminismo y la educación en y para nuestra América. Revista Venezolana de Estudios de la Mujer, $13(31), 17-26$

Goldsmith, M. (1998). Feminismo e investigación social. Nadando en aguas revueltas. En E. Bartra (Comp.) Debates en tomo a una metodología feminista (pp. 35-62.). México: Universidad Autónoma Metropolitana-Unidad Xochimilco 
Gordillo, A. M. (2004). ¿Qué es lo novedoso del método de investigación feminista? Encuentro, 70, 1-11 Recuperado de https://bit.ly/2WH2Gov

León, M. E. (2011). Ciencia, Tecnología y mujeres: una tríada disonante. Revista Filosofía Universidad de Costa Rica, $50(127-128), 155-161$

Longino, H. (1993). Subjects, Power and Knowledge: Description and Prescription. En L. Alcoff (Ed.), Feminist Philosophies of Science in Feminist Epistemologies. New York: Routledge.

Maffia, D. (2007). Epistemología feminista: La subversión semiótica de las mujeres en la ciencia. Revista Venezolana de Estudios de la Mujer, 12 (28), 63-98

Núñez, M. (2001). Los estudios de género en Cuba y sus aproximaciones metodológicas, multidisciplinarias y transculturales (1974-2001). IV Conferencia Internacional de la Cátedra de la Mujer de la Universidad de la Habana, Cuba. Conferencia llevada a cabo por la Universidad de la Habana, Cuba

OCDE (2015). The ABC of Gender Equality in Education: Aptitude, Behaviour, Confidence. París: PISA, OECD Publishing. Recuperado de http://dx.doi.org/10.1787/9789264229945-en

Ostrovsky, A. E. (2011). Epistemologías feministas: pensando en sus aportes a la reflexión crítica de la disciplina. Recuperado de https://bit.ly/2JFryJE

Schiebinger, L. (1993). Nature's Body: Gender in the Making of Modern Science. Boston: Beacon Press.

UNESCO (2017). Education transforms lives. Recuperado de https://bit.ly/1HxKuBo

Ungo, U. (2007). Las mujeres y la educación en américa latina: Una aproximación a los dilemas y desafíos. Revista Venezolana de Estudios de la Mujer, 12 (28), 205-220. Recuperado de https://bit.ly/2JDZJBt

Valdivieso, M. (2007). Críticas desde el feminismo y el género a los patrones de conocimiento dominantes. Revista Venezolana de Estudios de la Mujer, 12 (28), 185-22. Recuperado de https://dialnet.unirioja.es/servlet/articul o? codigo $=4136316$

\section{Notas}

[1] Pongo el énfasis en educar pues muchas veces se encierra la labor docente en el término enseñar. Considero que estamos convocados/as a trascender esa terminología y esforzarnos más en educar al alumnado, no solo en el tema que compete a este trabajo, sino en todas las facetas de la vida, a influir en que en tiempos tan complejos como los que vivimos les inculquemos valores sobre y para el bien común. No se trata de una utopía o de proponer políticas públicas generalizables, sino de que cada docente en su pequeño espacio -que es el salón de clases- tome conciencia de que la labor que ejecuta puede marcar diferencias. Basta un estudiante que formemos con mirada crítica hacia las desigualdades sociales vigentes, que sea capaz a futuro de reconocerlas y enfrentarlas, para que haya valido la pena el esfuerzo.

[2] Autoras, como Ana Elisa Ostrovsky en Epistemologias feministas: pensando en sus aportes a la reflexión crítica de la disciplina, se refieren al término en plural con el propósito de desmenuzar en el mayor grado posible las diferentes propuestas por períodos históricos. En el presente ensayo se utiliza el término en singular pues lo que interesa es evaluar los aportes del pensamiento feminista en un sentido general, que permita reflejar su utilidad cuando estudiamos el ámbito educativo desde y con una perspectiva de género.

[3] No empleo la x, por ejemplo al hablar de sujetos, pues en la dinámica académica de mi país se privilegian otras formas de denunciar las discriminaciones de género, no necesariamente quedan reflejadas en los términos que se utilizan para la elaboración de informes científicos. Esto no quiere decir que no esté consciente de la existencia de rasgos sexistas en el lenguaje científico, pero considero que a veces se abusa del uso de las x o las @.

[4] Utilizo el plural con el propósito de exponer presupuestos comunes sobre el tema, presentes en las diversas corrientes feministas.

[5] Otros autores en los que pueden observarse coincidencias al respecto son: Ungo (2007); Gargallo (2008), y Barffusón (et al., 2010).

[6] Lecturas imprescindibles para un mayor detenimiento en el tema las ofrecen Sandra Harding en el texto Feminism and Methodology, del año 1987 y D. Haraway en Ciencia, cyborgs y mujeres. La Reinvención de la Naturaleza, publicado en 1995.

[7] En términos generales, la diversidad de corrientes de pensamiento feministas. 
[8] Podrían citarse otros, como la diversidad de espacios que ocupa y formas en las que se expresa en la práctica el pensamiento feminista. Como afirma Barffusón, el "feminismo va de una movilización social y política hasta la elaboración teórico-crítica que se traduce en praxis social y política” (2010, p. 358).

[9] Herencia además de las escuelas segregadas, donde el sexismo era explícito. Una vez mixtas, no significó la desaparición de este, sino la creación de formas de expresión del sexismo más sutiles, pero no por ello menos eficaces.

[10] De hecho, autores como Acker y Barffusón señalan que muy pocas profesoras se percatan de la necesidad de emplear la perspectiva de género en el sistema educativo. Es decir, que no solo son los hombres dedicados a la docencia los que omiten la perspectiva de género, sino que muchas mujeres del sistema educativo no la perciben.

[11] Término identificado con el de enfoque de género y empleados indistintamente por numerosos autores, por lo que no constituye objetivo del presente trabajo distinguir sus especificidades conceptuales.

[12] Definidas por la UNESCO como innovadoras, efectivas, sostenibles y replicables.

[13] Sobre el tema resultó muy esclarecedor el texto de Mary Goldsmith Connelly citado con anterioridad, en tanto se hace hincapié en la importancia que le concede el feminismo como corriente crítica de pensamiento al contexto en el cual se genera el conocimiento y el producto mismo de ese proceso.

[14] Las ideas de Barffusón y otros autores resultaron de vital importancia al respecto, en tanto implementar la perspectiva de género en el ámbito educativo precisa de una evaluación cultural del significado de ser hombres o ser mujeres en nuestro propio contexto, de modo que prevalezcan los valores que contribuyan a nuestra realización plena como seres humanos.

[15] El estudio que propone el enfoque de género destaca la necesidad de analizar las realidades de género en relación con las de las clases sociales, las razas, y con todos los elementos presentes en las estructuras socioeconómicas en sociedades concretas en un momento histórico dado.

[16] En este espacio me gustaría incluir el término encasillamos pues inconscientemente puedo haber sido exponente de esta lamentable práctica educativa.

[17] Las lecturas al respecto sugieren que, si bien no existen marcadas diferencias entre las técnicas utilizadas en investigaciones desde la perspectiva feminista con las que se aplican en cualquier investigación androcéntrica, sí varía en gran medida la interpretación metodológica. No obstante, como herencias del pensamiento feminista y del empleo de la perspectiva de género, se proponen en la actualidad técnicas de investigación más participativas, indefiniendo un tanto la pared investigador/a-investigado/ a.

[18] Sobre el tema, son oportunos los criterios planteados por Francesca Gargallo. La autora hace referencia a "la elección y construcción de la validez de los conocimientos para sostener, posibilitar y reforzar los proyectos de los grupos que detentan el poder de gobierno, así como los medios para transmitirlos, hasta imponerlos como los únicos subsistentes" (2008, p. 19).

[19] Para el caso particular de Cuba, se ha logrado tomar conciencia de la importancia de aplicar la perspectiva de género en estudios de grado, lo que se ha traducido en experiencias positivas. Áreas como la Sociología o la Psicología, por citar solo dos ejemplos, se han visto beneficiadas con asignaturas dedicadas a los estudios de género incluidas en sus respectivos programas curriculares. Además, el Departamento de Sociología de la Universidad de la Habana cuenta con numerosas investigaciones al respecto y profesionales consolidadas en el tema. La Facultad de Psicología de dicho centro universitario cuenta con la Cátedra de Estudios de la Mujer, que ha transmitido durante años conocimientos y experiencias investigativas y prácticas docentes en relación directa con el enfoque de género. Existen otras cátedras y programas en Cuba dedicados a estos temas, lo que indica una toma de conciencia por parte de la academia de la necesidad de aplicar un enfoque inclusivo e incluyente en el medio educativo y en las prácticas docentes, no obstante, siempre quedan pasos por dar.

[20] En no pocos países se está evidenciando la tendencia de una mayor presencia femenina en algunos niveles del sistema educativo (Ungo, 2007).

[21] La perspectiva de género ofrece una mirada inclusiva a las realidades de hombres y mujeres, de hecho, es una de sus exigencias. No obstante, a los efectos de este trabajo se decidió enfatizar en las mujeres precisamente por la discriminación histórica que han sufrido sus experiencias al interior del sistema educativo formal e informal.

[22] En tal sentido, uno de los principales retos que enfrenta el sistema educativo en la región se evidencia en el caso de la mujer indígena. 
[23] Algunas autoras, como Urania Ungo, se cuestionan si es factible para muchas sociedades, cargadas de situaciones de pobreza, desigualdades y exclusión, continuar implementando políticas que no se corresponden con tales realidades y que, como efecto inesperado, contribuyen a agravar conflictos heredados.

[24] De gran importancia se nos revela la necesidad de tener en cuenta factores como la pobreza, exclusión étnica y marginalidad territorial como agravantes de las desigualdades de género en el sistema educativo. En este sentido, Graciela Messina ha evidenciado cómo las niñas de sectores más empobrecidos son las más excluidas del acceso a la educación.

[25] Término entendido no solo como la educación conjunta de hombres y mujeres, sino como un proceso intencionado que posibilite analizar y criticar las prácticas educativas y los contenidos que se imparten para reconocer y denunciar el sexismo en el medio educativo.

[26] Para ampliar sobre el tema puede consultarse el programa MOST1 Management of Social Transfotrmations de la UNESCO.

\section{BY-NC-ND}

